\title{
High-symmetry metal-organic frameworks as matrices for organic-based substitutional solid solutions
}

\author{
Fernando J. Uribe-Romo \\ Department of Chemistry \\ University of Central Florida \\ 4111 Libra Drive, Orlando, FL 32816 \\ fernando@ucf.edu
}

Preparation of stable, organic-based substitutional solid solutions (OSSS) with tunable properties is hindered, among other factors, by the lack of predictability of crystal packing of organic compounds. Their preparation would enable to access molecular properties observed in solution, but now in the crystalline solid state, and allow the creation of high precision organicbased materials for real-life applications. In this presentation we demonstrate, for the very first time, that high-symmetry, water stable, zirconia metal-organic frameworks, MOFs, can be utilized as matrices for OSSS of the organic links. High-symmetry cubic MOFs allow for an unprecedented level of synthetic control over the crystal structure of organic compounds, because of the low number of crystallographic degrees of freedom to control (only one lattice parameter), and their ability to integrate links of varied chemical functionality. We incorporated organic links of same metrics, yet with varied predetermined molecular activity (e.g., fluorescent or redox), into MOFs as OSSS at controllable ratios, enabling control of their concentration dependent traits as well as in their crystal packing. As a result, we prepared high-symmetry OSSS MOFs that exhibit phenomena such as dilute multifluorophore emission, excimer emission, and redox conductivity, in materials that are also stable to water and humidity with long shelf life. 